\title{
On the Mass Spectrum of Exotic Protonium Atom in Oscillator Representation Method
}

\author{
Arezu Jahanshir \\ Assistant professor and faculty member of Bueinzahra Technical University, Iran \\ jahanshir@bzte.ac.ir
}

\begin{abstract}
In the given paper one determines the constituent mass of the bound state and binding energy of hadronic exotic system, according to the basis investigation of the asymptotically behavior of the loop function of scalar particles in the external electromagnet field are analytically determined exploration of these states through relativistic and non-perturbative corrections. It is shown that, the mass spectrum of a relativistic bound state of protonium consisting from proton and anti-proton is differing from a mass of initial particles.
\end{abstract}

Keywords: protonium, constituent mass, Hamiltonian, Green function, scalar particles

\section{Introduction}

All of theoretical high energy physician by using mathematical methods and new theories try to understand how bound state arise in the formalism of quantum field theory and to work out effective methods to calculate all characteristics of these bound states, especially their masses, binding energy and spin interactions. The analysis of a bound state is difficult when the interaction have to be considered as relativistic, i.e. when they travel at speeds considerably near the " $c$ ". The theoretical criterion is that the coupling constant should be strong [1-6] and masses of intermediate particles gluons in quantum chromodynamic should be big in comparison with masses of constituents. New importance to the exotic hadronic bound state physics was given by the development of quantum chromodynamic, the modern theory of strong interactions. Thus, the definition relativistic corrections to the energy spectrum hadronic structures are one of the important problems for toddies. The technical achievements of experimental studies make it possible to obtain the exotic hadronic atoms with same particle with different charge one like protonium, quarkonium and etc. so interaction becomes the order of one, so that become strong and the calculation of relativistic corrections becomes necessary. An exotic two particles atom is look like a normal atom. Because these substitute particles are either unstable, exotic atoms have a short half-life. Protonium is an exotic hadronic atom which a proton and an antiproton orbit each other. Protonium is an electrically neutral boson with zero baryon number. The ATHENA experiment at CERN, after producing cold antihydrogen atoms for the first time in 2002, has synthesized protonium atoms in vacuum at very low energies. Protonium is of interest for testing fundamental physical theories. In the nested penning trap of the ATHENA apparatus protonium has been produced as result of a chemical reaction between an antiproton and the simplest matter molecule, $\mathrm{H}^{+}{ }_{2}$. The formed protonium atoms have kinetic energies in the range $40-700 \mathrm{MeV}$ and are metastable with mean lifetimes of the order of 0.1 to 10 microseconds. Theoretical studies of protonium have mainly used nonrelativistic quantum mechanics. These give predictions for the binding energy and lifetime of the states. Thus two or multi-particle interactions involving hadrons in intermediate states may be important. Hence the production and study of protonium would be of interest also for the understanding of inter-nucleon forces. For studying this system we must understand means of binding 
energy and mass spectrum. Binding energy is the mechanical energy required to disassemble a whole into separate parts. A bound system has a lower potential energy than its constituent parts; this is what keeps the system together. The usual convention is that this corresponds to a positive binding energy. In general, binding energy represents the mechanical work which must be done in acting against the forces which hold an object together, while disassembling the object into component parts separated by sufficient distance that further separation requires negligible additional work. Binding energy is a measure of the energy required to free particle from their exotic atomic orbits. At the atomic level, the atomic binding energy of the hadronic atom derives from electromagnetic/ strong interaction [4-9] and is the energy required to disassemble an atom into particle and an anti-particle. The author in this article propose one of the versions of calculation of the mass spectrum and binding energy of bound state systems in the framework of quantum field theory the expression that takes into account strong interaction corrections to the mass of the bound state with a known potential is proposed on the basis of calculating the asymptotic behavior of correlation functions.

\section{General Theoretical Discussion}

This work is devoted to study the bound states problems, which will be carried out on the research basis asymptotical behavior of two scalar particles in external gauge field. In this case, loop function of two scalar particles with different masses $m_{1}$ and $m_{2}$ with average on external statistical field is considered and the Green function is used. The polarization operator in an external electromagnetic field looks like [10-13]:

$\Pi(x-y)=\left\langle G_{m 1}(x, y \mid A) G_{m 2}^{*}(y, x \mid A)\right\rangle_{A}$.

The Green function $G(x, y \mid A)$ for scalar particles in an external field is determined from the equation:

$$
\left[\left(i \frac{\partial}{\partial x_{a}}+\frac{g}{c \hbar} A_{a}(x)\right)^{2}+\frac{c^{2} m^{2}}{\hbar^{2}}\right] G(x, y \mid A)=\delta(x-y),
$$

where $m$ is the mass of a scalar particle, and $g$ is the coupling constant of interaction.

The mass of the bound state is determined by the equation [11]:

$M=-\lim _{|x-y| \rightarrow \infty} \frac{\ln \Pi(x-y)}{|x-y|}$.

After averaging over the field $A_{\alpha}(x)$ we have (for details of an evaluation see [12-16]):

$\Pi(x)=\int_{0}^{\infty} \int_{0}^{\infty} \frac{d u_{1} d u_{2}}{\left(8 x \pi^{2}\right)^{2}} \exp \left\{-\frac{x}{2}\left(\frac{m_{1}^{2}}{\mu_{1}}+\mu_{1}\right)-\frac{x}{2}\left(\frac{m_{2}^{2}}{\mu_{2}}+\mu_{2}\right)\right\} \cdot J_{\mu}\left(\mu_{1}, \mu_{2}\right)$,

where:

$$
\begin{gathered}
J\left(\mu_{1}, \mu_{2}\right)=N_{1} N_{2} \iint \delta \vec{r}_{1} \delta \vec{r}_{2} \exp \left\{-\frac{1}{2} \int_{0}^{1 x} d \tau\left(\mu_{1} \dot{\vec{r}}_{1}^{2}(\tau)+\mu_{1} \dot{\vec{r}}_{2}^{2}(\tau)\right)\right\} . \\
\cdot \exp \left\{-W_{1,1}+W_{1,2}-W_{2,2}\right\}
\end{gathered}
$$


Functional integral in (5) is similar to the Feynman Path integral trajectories in nonrelativistic quantum mechanics [12-17] for the motion of two particles with masses $\mu_{1}$ and $\mu_{2}$. The interaction of these particles is described by the nonlocal functional $W_{i j}$, in which are contained both potential and non-potential interaction.

Taking into account (4) and (5) in the limit $|x-y| \rightarrow \infty$ from (3) for the mass of the bound state we get (for detail see [10-17]):

$M_{\text {system }}=\sqrt{m_{1}^{2}-2 \mu^{2} \cdot \frac{\partial E(\mu)}{\partial \mu}}+\sqrt{m_{2}^{2}-2 \mu^{2} \cdot \frac{\partial E(\mu)}{\partial \mu}}+\mu \cdot \frac{\partial E(\mu)}{\partial \mu}+E(\mu)$,

where the parameter $\mu$ is determined from the equation:

$\frac{1}{\mu}=\frac{1}{\mu_{1}}+\frac{1}{\mu_{2}}=\frac{1}{\sqrt{m_{1}^{2}-2 \mu^{2} \cdot \frac{\partial E(\mu)}{\partial \mu}}}+\frac{1}{\sqrt{m_{2}^{2}-2 \mu^{2} \cdot \frac{\partial E(\mu)}{\partial \mu}}}$

and $E(\mu)$ - is the eigenvalue of the nonrelativistic Hamiltonian. Parameters $\mu_{1}$ and $\mu_{2}$ are mass components of the bound state (constituent mass of particles), which are different from the masses $m_{1}, m_{2}$ of free condition.

\section{Exotic Hadronic Atom: Protonium}

At this paragraph based on the described method on the above paragraph for proton and antiproton particles in external gauge field. In this case, loop function of two scalar particles with masses $m$ from equation (1) [10]:

$\Pi(x-y)=\left\langle G_{m}(x, y \mid A) G_{m}^{*}(y, x \mid C)\right\rangle_{A}$.

The Green function $G(x, y \mid A)$ for scalar particles in an external field is determined from the equation:

$\left[\left(i \frac{\partial}{\partial x_{a}}+\frac{g}{c \hbar} A_{a}(x)\right)^{2}+\frac{c^{2} m^{2}}{\hbar^{2}}\right] G(x, y \mid A)=\delta(x-y)$,

After averaging over the field $A_{\alpha}(x)$ from (9) we have:

$\Pi(x)=\int_{0}^{\infty} \int_{0}^{\infty} \frac{d u_{1} d u_{2}}{\left(8 x \pi^{2}\right)^{2}} \exp \left\{-x\left(\frac{m^{2}}{\mu}+\mu\right)\right\} \cdot J_{\mu}(\mu)$,

where:

$J(\mu)=N_{1} N_{2} \iint \delta \vec{r}_{1} \delta \vec{r}_{2} \exp \left\{-\frac{1}{2} \int_{0}^{1 x} d \tau\left(\mu \dot{\vec{r}}_{1}^{2}(\tau)+\mu \dot{\vec{r}}_{2}^{2}(\tau)\right)\right\} \cdot \exp \left\{-W_{1,1}+W_{1,2}-W_{2,2}\right\}$.

for the mass of the bound state we get (for detail see [11-13]):

$M_{P \tilde{P}}=2 \sqrt{m^{2}-2 \mu^{2} \cdot \frac{\partial E(\mu)}{\partial \mu}}+\mu \cdot \frac{\partial E(\mu)}{\partial \mu}+E(\mu)$,

where the parameter $\mu$ is determined from the equation: 
$\frac{1}{\mu_{P}}=\frac{2}{\mu}=\frac{2}{\sqrt{m^{2}-2 \mu^{2} \cdot \frac{\partial E(\mu)}{\partial \mu}}}$

and $E(\mu)$ - is the eigenvalue of the nonrelativistic Hamiltonian. Parameter $\mu$ is mass components of the bound state (constituent mass of particles), which are the masses $m$ of free condition. Now as we know one of the basic problems of nonrelativistic and relativistic quantum mechanics is to determine the energy spectrum and eigenvalue of the Hamiltonian of system described by the Schrödinger equation with a special potential. Complete solution of this equation has been determined for the Coulomb potential. It is well known that for determining the mass of the bound state systems first of all one should determine the eigenvalue of the Hamiltonian with Coulomb potential in protonium exotic system. For this aim we consider the following Schrödinger equation:

$\left[\frac{\vec{P}^{2}}{2 \mu}-\alpha r^{-1}\right] \Psi=E(\mu) \Psi$

$\alpha$ - is the coupling constant of electromagnetic interaction. And this equation for the wave function of orbital excitation $\left(\hat{\ell}^{2}=\ell(\ell+1)\right)$ is [18-21]:

$\left[\frac{-1}{2 \mu} \cdot\left[\frac{\partial^{2}}{\partial r^{2}}+\frac{2}{r} \frac{\partial}{\partial r}-\frac{\hat{\ell}}{r^{2}}\right]-\frac{\alpha}{r}\right] \Psi=E(\mu) \Psi$.

And at large distance, for the Coulomb potential the asymptotic behavior of wave function is well known [18-20].

We have to modify the variables in the starting Schrödinger equation. In this case, we used the substitution $r=q^{2 \rho}$, and found the following conditions:

$$
\begin{aligned}
& \frac{\partial^{2}}{\partial r^{2}}=\frac{q^{2-4 \rho}}{4 \rho^{2}}\left[\frac{\partial^{2}}{\partial q^{2}}+\frac{1-2 \rho}{q} \frac{\partial}{\partial q}\right] \\
& \frac{\partial}{\partial r}=\frac{1}{2 \rho} q^{1-2 \rho} \frac{\partial}{\partial q} \\
& \frac{\partial^{2}}{\partial r^{2}}+\frac{2}{r} \frac{\partial}{\partial r}=\frac{q^{2-4 \rho}}{4 \rho^{2}}\left[\frac{\partial^{2}}{\partial q^{2}}+\frac{1-2 \rho}{q} \frac{\partial}{\partial q}\right]
\end{aligned}
$$

After simple calculations we obtain Schrödinger equation in oscillator representation method (for the Coulomb potential $\rho=1$ ):

$\left[\frac{-1}{2 \mu} \cdot\left[\frac{q^{2}}{4}\left[\frac{\partial^{2}}{\partial q^{2}}-\frac{1}{q} \frac{\partial}{\partial q}\right]-\frac{\hat{\ell}^{2}}{q^{2}}\right]-\frac{\alpha}{q^{2}}\right] \Psi=E(\mu) \Psi$,

As a result in the zero approximation of oscillator representation, we have determined the Coulomb energy spectrum of hydrogen atom as follow:

$E(\mu)=-\frac{\alpha^{2}}{2(\ell+1)^{2}} \mu$,

The results of the zero approximation of this method are in good agreement with the exact values. Now let us to determine the mass spectrum of hadronic system with masses $m$. In this case for the binding energy we have:

$E_{b i n}=M-m$ 
where $E_{\text {bin }}$-is the bound state energy.

After some simplification from (2-5) for the mass of protonium system with $\ell=0[20$ 24] get:

$M_{P \tilde{P}}=m_{P} \cdot \sqrt{4-\alpha^{2}}$

and the constituent mass of each proton is:

$\mu_{P}=\frac{2 m_{P}}{\sqrt{4-\alpha^{2}}}$,

The bound state energy of exotic hadronic atom system (protonium) with coulomb strong interaction is

$E_{\text {bin }}=m_{P} \cdot\left(\sqrt{4-\alpha^{2}}-2\right)$

One determines final results for protonium system with $m_{p}=938.254 \mathrm{MeV}$ show in Table 1.

Table 1. The Constituents Mass, the Mass Spectrum, the Bound State
Energy for the Protonium Without Accounting Spins Interactions

\begin{tabular}{|c|c|c|c|}
\hline$\alpha$ & $\mu_{P(\mathrm{MeV})}$ & $M_{P \tilde{P}(\mathrm{MeV})}$ & $-E_{\text {bin }(\mathrm{MeV})}$ \\
\hline 0,015 & 938.725 & 1875.573 & 0.938 \\
\hline 0,015 & 938.725 & 1875.573 & 0.938 \\
\hline 0,142 & 940.607 & 1871.820 & 4.691 \\
\hline 0,259 & 945.823 & 1861.499 & 15.012 \\
\hline 0,500 & 969.272 & 1816.464 & 59.920 \\
\hline
\end{tabular}

\section{Conclusion}

In this paper, we studied one of the exotic hadronic atom systems on the basis of investigation of asymptotic behavior of the loop function for the scalar particles in the external gauge field and determined the mass spectrum and binding energy with strong feature of interaction. We have been able to obtain the constituent mass of protonium system. It is shown that the mass of particles is different in bound and free states. We have proposed a method for computing the bounding energy, mass spectrum and constituent mass for quantum systems with relativistic character of interaction. The calculation is based on the oscillator representation method and modifies Schrodinger equation. We have found out that determining the mass of the bound state systems requires; first of all, determining the Eigenvalue of the Hamiltonian with Coulomb potential and then calculating the mass and binding energy of hadronic system which we could achieve. This method provides us with magnificent information comparing to other theories such as perturbative and variation. The current paper has calculated energy spectrum and hadronic atom constituents mass in limited core mass conditions and we could conclude that constituents mass in the system is different from mass in free conditions. The method presented in this paper shows that results which we can be easily used for all exotic atoms with known potential and for orbital quantum number. The results are showing that the constituent mass will decrease with growth of the bounding energy the protonium system will stabile with growth of the coupling constant. 


\section{References}

[1] H. D. Politzer, Phys. Rev. Lett, vol. 30, no. 1346, (1973).

[2] M. G. Boshier and P. E. G. Baird and C. J. Footetal, Phys. Rev. A, vol. 40, no. 6169, (1989).

[3] W. Greiner and Y. Reinhort, "Quantum Electrodynamics", Springer- Verleg, (1992).

[4] E. C. Abers and B. V. Li, "Theory of Colibration", Mir, Moscow, pp. 241, (1977).

[5] Y. Lucha and F. F. Schober, "Spinless Salpeter Equation: Analytic Re-salts", Proceeding of the XI International Conference, Dubna, Russia, (1998).

[6] M. G. Boshier, P. E. Baird, C. J. Foot, E. A. Hinds, M. D. Plimmer, D. N. Stacey, J. B. Swan, D. A. Tate, D. M. Warrington and G. K. Woodgate, Phys. Rev., A 40, 6169, (1989).

[7] T. Udem and A. Huber, Phys. Rev. Lett., vol. 79, no. 2646, (1947).

[8] F. G. Mariam and W. Beer, Phys. Rev. Lett., vol. 49, no. 993, (1982).

[9] W. Liu and M. G. Boshier, Phys. Rev. Lett., vol. 82, no. 711, (1999).

[10] M. Dineykhan, G. V. Efimov and Kh. Narmsrai, Fortsh. Phys., vol. 39, no. 259, (1991).

[11] R. P. Feynman and A. P. Hibbs, "QMs and Path Integrals", NY McGraw-Hill, (1965).

[12] M. Dineykhan, A. Jahanshir and S. K. Sakhyev, Sci. Journal. Poisk, vol. 3, no. 199, (2003).

[13] M. Dineykhan, A. Jahanshir, D. S. Ashimov and S. Sakhyev, "On the Structure of QCD String", Proceeding of the Second Eurasian Conference on Nuclear Science and ITS Application, Almaty, Kazakhstan, (2002).

[14] N. Fröman and O. Fröman, "Physical Problems Solved by the Phase-integral Method", Cambridge Uni. Press, (2002).

[15] L. D. Landau and E. M. Lifshitz, "QMs: Non-Relativistic Theory”, Pergamon Press, (1977).

[16] L. Bergmann, C. Schaefer and W. Raith, "Constituents of Matter: Atoms, Molecules, Nuclei and Particles", Berlin: Walter de Gruyter, (1997).

[17] M. Dineykhan and G. V. Efimov, "On Bound States in the Quantum Field Theory", Proceeding of the First International Sakharov Conference on Physics, Moscow, USSR, (1991) May 27-31.

[18] M. Dineykhan and G. V. Efimov, Reports on Mathematical Physics, vol. 36, no. 287, (1995).

[19] M. Dineykhan and R. G. Nazmitdinov, Journal Yad. Fiz., vol. 62, no. 143, (1999).

[20] A. Jahanshir, Proceeding of the First International Conference on Physics, Tehran, Iran, (2004) January $1-4$.

[21] M. Dineykhan, S. Zhaugasheva, N. Toinbaeva and A. Jahanshir, Journal of Physics B: Atomic, Molecular and Optical Physics, vol. 42, no. 14, 145001, (2009).

[22] A. Jahanshir, Journal of Theoretical and Applied Physics, vol. 3, no. 4, 10, (2010).

[23] A. Jahanshir, PhD Thesis, National Library, Almaty, Kazakstan, (2003).

[24] M. Dineykhan, A. Jahanshir and C. Sakheev, Sci. Journal Poisk, vol. 3, no. 2, (2003), pp. 203. 